\title{
Artifact Correction for Zernike Phase-Plate Cryo-Electron Tomography
}

Haixin Sui ${ }^{1,2}$, Gregory Kishchenko ${ }^{1}$, Radostin Danev $^{3,4}$, Jie He ${ }^{1}$, Rebecca Fisher ${ }^{1}$, Chyongere Hsieh ${ }^{1}$ and Michael Marko ${ }^{1}$

1. Wadsworth Center, New York State Department of Health, Albany, NY 12201

2. Dept. of Biomedical Sciences, School of Public Health, University at Albany, Albany, NY 12201

3. Okazaki Institute for Integrative Bioscience, National Institutes of Natural Sciences, Okazaki, Japan

4. Max-Planck-Institute for Biochemistry, Martinsried 82152, Germany

In cryo-electron microscopy (cryo-EM), vitreously frozen biological specimens can tolerate only a limited electron dose for imaging. Therefore, cryo-EM micrographs display weak contrast and a poor signal-to-noise ratio. This limits data interpretability and prevents efficient application of cryo-EM to a wider range of biological problems. Continued efforts have been invested in imaging methods using phase plates for cryo-EM, as this technique can offer greatly enhanced image contrast [1-3]. While various types of phase-plate are under development, Zernike-type phase-plate imaging has been successfully applied to a number of cryo-EM studies in both single-particle and electron tomographic studies [4-9].

With typical Zernike-type phase plates, the abrupt edge of the phase-plate central hole (at the so-called "cut-on") results in fringes surrounding high-contrast objects and contrast inversion at the middle of large objects [10]. Based on simulation, it is suggested that the suitable object size for Zernike-phase-contrast imaging should be smaller than half of the cut-on periodicity [11]. Such a requirement limits the application of Zernike phase-contrast imaging in cryo-electron tomography which generally involves larger-sized objects.

Using Zernike phase-contrast imaging, we carried out a tomographic study of vitreously frozen radial spokes associated with microtubule doublets from Chlamydomonas. The radial spoke is an important structural component in the axoneme and regulates the beating motion of motile cilia and flagella. Its structure has been studied by cryo-electron tomography using defocus phase-contrast imaging [12,13]. The structural map displays a T-shaped configuration, with a ring-like "head" towards the central-pair complex at the center of the axoneme, and a long "stalk" attached to the peripheral microtubule doublets. The length of the head and stalk together is about $43 \mathrm{~nm}$. In our study, we used a Zernike phase plate with a cut-on periodicity of $43 \mathrm{~nm}$, the same as the length of the radial spoke.

Zernike phase-contrast imaging significantly improved micrograph contrast compared to that obtained by defocus-phase-contrast imaging [14]. Tomographic tilt series images were aligned and reconstructed. In the resultant tomogram, arrays of radial spokes complexes are clearly visible on the microtubule doublets without any image processing or contrast enhancement. The structural map of radial spokes was obtained by sub-volume averaging. The map agrees very well with the results published previously $[13,15]$. However, the fringes caused by the cut-on of the Zernike phase plate introduced obvious artifacts in the density map. We have used two methods of de-fringing to correct the artifacts in the micrographs of the tomographic tilt series. Both methods corrected the artifact to our satisfaction. Our results demonstrate that fringe reduction by image processing can help to correct artifacts in the structural maps, and is important in cryo-electron tomography using Zernike phase-contrast imaging. 


\section{References}

[1] R Danev and K Nagayama, Ultramicroscopy 99 (2001), p. 243.

[2] J Shiue et al., J. Elect. Microsc. 58 (2009), p. 137.

[3] R Cambie et al., Ultramicroscopy 107 (2007), p. 329.

[4] R Danev and K Nagayama, J. Struct. Biol. 161 (2008) p. 211.

[5] H Shigematsu et al., J. Biol. Chem. 285 (2010) p. 11210.

[6] K Murata et al., Structure 18 (2010), p. 903.

[7] R Rochat et al., J. Virol. 85 (2011), p. 1871.

[8] W Dai et al., Nature 502 (2013), p. 707.

[9] RC Guerrero-Ferreira and ER Wright, J. Struct. Biol. 185 (2014), p. 129. [10] R Danev et al., Ultramicroscopy 111 (2011), p. 1305.

[1 1] R Danev et al., Ultramicroscopy 109 (2009), p. 312.

[12] CF Barber et al., Mol. Biol. Cell 23 (2012), p. 111.

[13] G Pigino et al., J. Cell Biol. 295 (2011), p. 673.

[14] H Sui and KH Downing, Nature 442 (2006), p. 475.

[15] J Lin et al., Cytoskeleton (Hoboken) 69 (2012), p. 88.

[16] Supported by NIH grants GM101026 (H. Sui) and GM103555 (M. Marko). 